\title{
MULTIDRUG RESISTANCE AMONG ENTEROCOCCI AT A TERTIARY CARE HOSPITAL IN NORTHERN BULGARIA
}

\section{Valentina P. Popova, Mariya P.Sredkova, Hristina H. Hitkova, Kaloyan T. Ivanov', Vladimir G. Popov ${ }^{2}$}

Sector of Microbiology and Virology, Department of Microbiology, Virology and Medical Genetics, Medical University - Pleven

${ }^{1}$ Student, Medical University - Pleven

${ }^{2}$ Department of Propaedeutics Internal Diseases, Medical University-Pleven, Bulgaria

\section{Corresponding Author:}

Valentina P. Popova

Sector of Microbiology and Virology, Medical University

1. St. Kliment Ohridski str.

Pleven, 5800

Bulgaria

e-mail:hitkova@abv.bg

Received: March 14, 2013

Revision received: April 15, 2013

Accepted: June 26, 2013

\begin{abstract}
Summary
Multidrug-resistant (MDR) enterococci are a growing threat. The aim of this study was to determine the species distribution and prevalence of multidrug resistance among 100 enterococcal strains, isolated from patients treated in the University Hospital in Pleven, Bulgaria. Susceptibility to 11 antimicrobial agents was determined, using the disc diffusion method according to the performance standards of Clinical Laboratory Standards Institute (CLSI), 2012. All isolates were screened for high-level aminoglycoside resistance and resistance to vancomycin according to the recommendations of CLSI, 2012. For strains with reduced susceptibility to vancomycin, minimal inhibitory concentrations (MIC) of glycopeptides were determined by Etest (Liofilchem, Italy) and by Vitek 2 automated system. Our results demonstrated decreased susceptibility of enterococci to almost all intensively used anti-enterococcal drugs. Resistance to both penicillins (ampicillin and penicillin) among E.faecium strains was significantly higher (83$87 \%$ ) than among E.faecalis isolates (4-27\%). HLGR was detected in $70 \%$ of E.faecium and $38 \%$ of E.faecalis isolates. All HLGR strains were found to be multiple-drug resistant. Of particular note was the emergence of concomitant resistance to 6 antimicrobials in almost $50 \%$ of E.faecium isolates. Despite the wide dissemination of MDR E.faecium strains penicillins in our hospital, acquired resistance to vancomycin was not found.

Key words: enterococci, antimicrobial resistance
\end{abstract}

\section{Introduction}

Enterococci are resistant to a wide range of antimicrobial agents. This feature allows them to survive in hospital environment, where antibiotics are used, and favours the dissemination of resistant organisms $[1,2]$. During the last few years, the prevalence of multidrug-resistant enterococci has been steadily increasing around the world, and remarkable changes in the epidemiology of enterococcal infections have been documented. Increasing rates of vancomycin-resistant enterococci (VRE) in several European countries are due to an increasing prevalence of vancomycin- 
resistant E.faecium. Defined clonal groups of E.faecium show an enhanced capacity to disseminate in nosocomial setting and are thus called epidemic or hospital-acquired [3, 4]. Such strains are mostly ampicillin-resistant, partly high-level ciprofloxacin-resistant, and possess additional virulence traits [3-7]. Multiple-drug resistance is attributable but not limited to vancomycin-resistant enterococci [8]. Colonization and infection with MDR enterococci occur worldwide. The literature suggests that spread of ampicillin resistant, hospital-acquired E.faecium strains is a pre-requisite for successful establishment of VRE and further dissemination of vancomycin resistance among the hospital E.faecium population in general [3]. Despite the increasing number of reports on VRE in many countries, vancomycin resistance is very rare in Bulgarian hospitals [9]. Only a few vancomycinresistant enterococcal strains have been reported so far [10]. The present study was conducted to achieve early detection of emerging resistance.

The aim of this study was to determine the species distribution and prevalence of multidrug resistance among 100 enterococcal strains, isolated from patients treated in the University Hospital in Pleven, Bulgaria.

\section{Materials and Methods}

\section{Bacterial strains}

A total of 100 enterococcal isolates were investigated. The strains were isolated from clinical specimens of patients admitted to the University Hospital in Pleven between January and April 2012. Duplicate isolates from the same patient were excluded. Enterococcal strains were collected from urine (66), wound specimens (24), aspirates (5), blood (2) and other specimens (3).

\section{Identification}

Identification of enterococci to the species level was based on series of conventional physiological tests $[11,12]$ and Vitek 2 automated system (bioMerieux, France).

\section{Antimicrobial susceptibility testing}

Susceptibility to penicillin, ampicillin, gentamicin, ciprofloxacin, erythromycin, tetracycline, nitrofutantoin, vancomycin, teicoplanin, tigecycline (BBL, Becton Dickinson, USA) and linezolid (Oxoid ltd, UK) was determined by the disc diffusion method according to the performance standards of Clinical Laboratory
CLSI), 2012 [13].

\section{Beta-lactamase production}

All ampicillin resistant strains were tested for beta-lactamase production with the chromogenic nitrocefin disc test (BBL, Becton Dickinson, USA).

\section{Screening for high-level amino- glycoside resistance}

High-level aminoglycoside resistance was determined by disc diffusion method using a disc of $120 \mu \mathrm{g}$ gentamicin.

\section{Screening for vancomycin resistance}

All isolates were screened for resistance to vancomycin, using a screening agar containing $6 \mu \mathrm{g} / \mathrm{ml}$ vancomycin, as recommended by CLSI [13]. For strains with reduced susceptibility to vancomycin, minimal inhibitory concentrations (MIC) of glycopeptides were determined by $\mathrm{E}$ test (Liofilchem, Italy), as well as by Vitek 2 automated system. Results were interpreted according to the recommendations of CLSI [13].

\section{Multidrug resistance}

Multidrug resistance was defined as resistance to three or more classes of antimicrobial agents.

\section{Results}

Out of 100 enterococcal isolates, 73 were identified as E.faecalis and 23 - as E.faecium. The remaining enterococcus species were presented by E.gallinarum (3\%) and E.raffinosus $(1 \%)$.

The antimicrobial resistance patterns of isolated enterococci are shown in Table 1. Resistance to penicillin and ampicillin was found in $40 \%$ and $21 \%$ of all tested isolates, respectively. Resistance to both penicillins among E. faecium strains was significantly higher (83-87\%) than among E.faecalis isolates (4$27 \%$ ). None of isolates produced beta-lactamase.

HLGR was detected in almost half (44\%) of the isolates. E.faecium showed higher resistance rate $(70 \%)$ than E.faecalis (38\%). All HLGR strains were found to be multiple-drug resistant. Out of 44 high-level gentamicin resistant strains, $35(79.55 \%)$ and $18(40.91 \%)$, were resistant to penicillin and ampicillin, respectively and more than $90 \%(93.18 \%)$ showed concomitant resistance to ciprofloxacin and erythromycin.

Comparative data about antimicrobial 
Table 1. Resistance to antimicrobial agents among clinical enterococcal isolates

\begin{tabular}{lllll}
\hline \multirow{2}{*}{ Antimicrobial agents } & \multicolumn{4}{c}{ Number (\%) of resistant strains } \\
\cline { 2 - 5 } & $\begin{array}{l}\text { E. faecalis } \\
\mathrm{n}=73\end{array}$ & $\begin{array}{l}\text { E. faecium } \\
\mathrm{n}=23\end{array}$ & $\begin{array}{l}\text { Other enterococci } \\
\mathrm{n}=4\end{array}$ & $\begin{array}{l}\text { Total } \\
(\mathrm{n}=100)\end{array}$ \\
\hline Penicillin & $20(27.40 \%)$ & $20(86.96 \%)$ & 0 & $40(40 \%)$ \\
\hline Ampicillin & $3(4.11 \%)$ & $19(82.61 \%)$ & 0 & $21(21 \%)$ \\
\hline Gentamicin (HLR) & $28(38.36 \%)$ & $16(69.57 \%)$ & 0 & $44(44 \%)$ \\
\hline Ciprofloxacin & $36(49.32 \%)$ & $21(91.30 \%)$ & 1 & $58(58 \%)$ \\
\hline Erythromycin & $61(83.56 \%)$ & $22(95.65 \%)$ & 2 & $85(85 \%)$ \\
\hline Tetracyclin & $64(87.67 \%)$ & $3(13.04 \%)$ & 2 & $69(69 \%)$ \\
\hline Vancomycin & 0 & 0 & $3 *$ & $3 *(3 \%)$ \\
\hline Teicoplanin & 0 & 0 & 0 & 0 \\
\hline Linezolid & 0 & 0 & 0 & 0 \\
\hline Tigecyclin & 0 & 0 & 0 & 0 \\
\hline Nitrofurantion & 0 & $14(60.87 \%)$ & 0 & $14(14 \%)$ \\
\hline
\end{tabular}

* Strains with reduced susceptibility to vancomycin

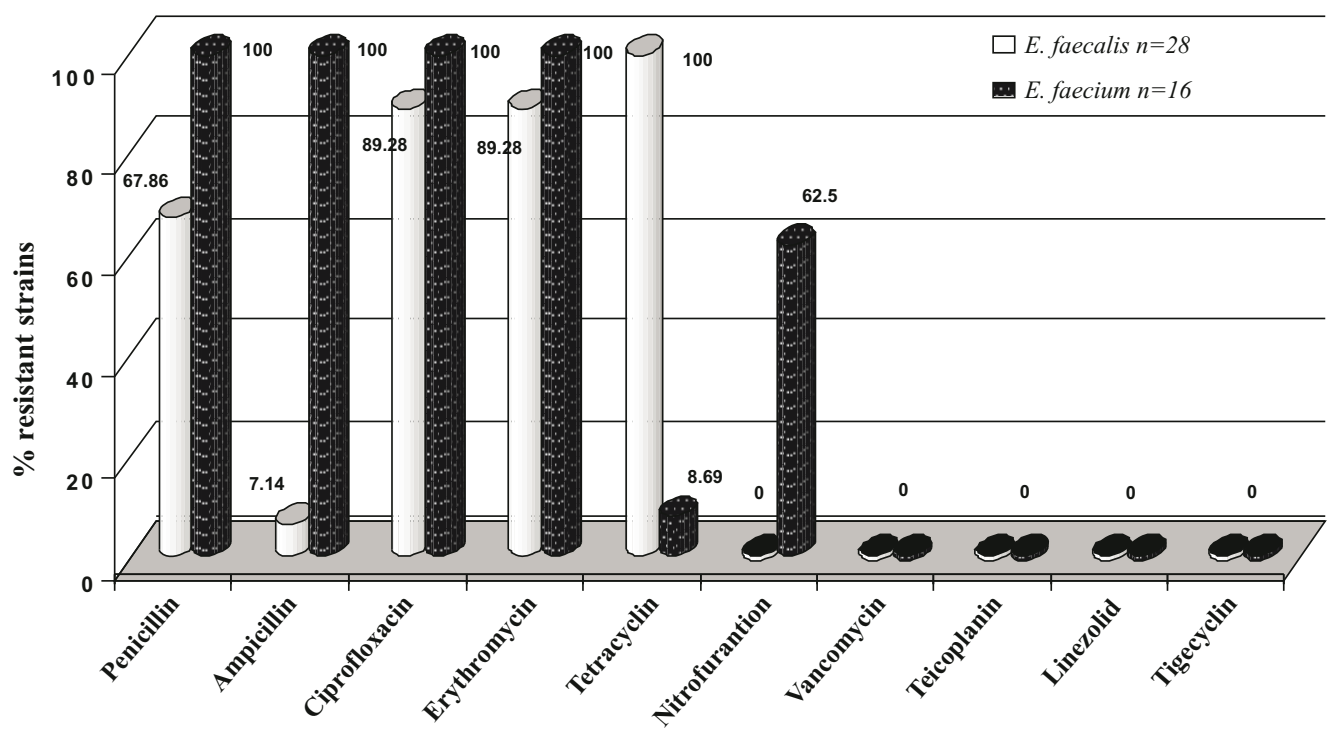

Figure 1. Resistance to antimicrobial agents among HLGR E.faecalis and E.faesium strains

resistance of HLGR E.faecium and E.faecalis strains are shown in Figure 1. The HLGR E.faecium strains demonstrated higher resistance rates to penicillin, ampicillin and nitrofurantoin than E.faecalis $(\mathrm{p}<0.01)$. Both species showed similarly high resistance rates $(\mathrm{p}>0.05)$ to ciprofloxacin and erythromycin (89\% and $100 \%$, respectively). All HLGR E.faecalis isolates were resistant to tetracycline, but they were fully susceptible to nitrofurantoin.
MDR was established in $78 \%$ of E.faecium and $37 \%$ of E.faecalis strains. The concomitant resistance to 6 antimicrobials was found in almost $50 \%$ of $E$. faecium isolates.

Three isolates were considered to be putative vancomycin-resistant enterococci, upon their growth on the screening medium with $6 \mu \mathrm{g} / \mathrm{ml}$ of vancomycin. Testing the susceptibility to glicopeptydes revealed that all three strains had intermediate vancomycin $\mathrm{MIC}_{\mathrm{s}}(6-8 \mu \mathrm{g} / \mathrm{ml})$, and 
were susceptible to teicoplanin $\left(\mathrm{MIC}_{\mathrm{s}}\right.$ of 0.5 $\mu \mathrm{g} / \mathrm{ml})$. All strains with reduced susceptibility to vancomycin were identified as E.gallinarum. Therefore, acquired vancomycin resistance was not detected. All enterococcal isolates were susceptible to teicoplanin, linezolid and

\section{Discussion}

Enterococci are important nosocomial pathogens and their growing resistance to most of the clinically relevant antibiotics makes them a major threat. The results of this study demonstrate decreased susceptibility of enterococci to almost all intensively used antimicrobial agents. Our data confirmed significant differences between the antimicrobial resistance patterns of enterococcal species. Only glycopeptides, linesolid and tigecycline were highly effective against both species of enterococci. Ampicillin and nitrofurantoin showed good activity only against E.faecalis, while tetracycline was effective against $E$. faecium strains.

Penicillin and ampicillin are first-line therapeutic agents for treatment of enterococcal infections. According to reports from different parts of Europe, ampicillin resistance among E.faecalis varies between 0 and 17\% [14-16]. Similar low rate of resistance $(4 \%)$ was established in our institution. In contrast, resistance of E.faecium to ampicillin has increased significantly during the last years, due to the wide dissemination of ampicillin-resistant strains that belong to the polyclonal subcluster $\mathrm{CC}_{17}$ [17]. According to the Tigecycline Evaluation and Surveillance Trial [18] performed in the major European countries between 2004 and 2009 , the resistance to ampicillin among E. faecium ranged from $72.9 \%$ (France) to $93.3 \%$ (Germany). Our data confirmed this trend. In the present study, more than $80 \%$ of E.faecium strains were resistant to penicillin and ampicillin. Interestingly, all ampicillin resistant strains demonstrated concomitant resistance to ciprofloxacin. This is in agreement with a Swedish colonization study, conducted by Torell et al. [19], who showed that $91 \%$ of ampicillin resistant strains were concomitantly resistant to ciprofloxacin. They demonstrated that ampicillin resistant enterococci carriage rates correlated with the use of fluoroquinolones. In our study, an increased resistance of Enterococcus spp. to ciprofloxacin, particularly E.faecium $(91 \%)$ was found. This high-level resistance could be associated with the excessive and uncontrolled use of fluoroquinolones in our country. Another possible explanation might be an exceptional capacity for epidemic spread of certain clones with this resistance pattern [20]. It is known that acquired ampicillin and high-level ciprofloxacin resistance appear as good phenotypic markers of hospital-acquired E.faecium strains [3, 4, 21, 22].

A growing resistance of enterococci to aminoglycosides is observed worldwide. In a study performed in 27 European countries during 1999 [23], HLGR in E.faecium was as frequent as in E.faecalis ( $23 \%$ and $20 \%$, respectively). On the contrary, such resistance was not detected among E.faecium strains, isolated in eight Swedish hospitals [24]. In contrast to previously reported results by Sredkova [25], present data indicate that HLGR among E.faecium isolates $(70 \%)$ is higher than in E.faecalis $(38 \%)$. This fact is due to the wide dissemination of E. faecium strains in our hospital. According to the data of EARS [17] HLGR in E.faecalis seems stable in Europe but at relatively high levels (between $25 \%$ and $50 \%$ ). Our resistance rate in respect to E.faecium was close to that reported in Thailand [26] and Turkey [27], (76\% and 88\%, respectively) but higher than in Saudi Arabia [28] where only $18.5 \%$ of strains were resistant. This high-level resistance to aminoglycosides, observed in both species in our study is a real problem because it could nullify the synergistic effect of combined therapy with cell-wall active agents, recommended for the treatment of serious enterococcal infections. Moreover, these strains were also resistant to other antibiotics, and this resistance additionally limits therapeutic options.

Significant correlation between enterococcal species and resistance to tetracycline and nitrofurantoin was observed in our study. Surprisingly, we found that approximately $90 \%$ of E.faecalis strains were resistant to tetracycline, as compared to only $13 \%$ of E.faecium. Similar results have previously been observed in enterococci isolated from food [29]. However, Quinones [30] has established high resistance rates $(78-90 \%)$ in both species. It has generally been reported that resistance to nitrofurantoin is low $[30,31]$. Susceptibility to Nitrofurantoin was found to be $100 \%$ for the .E.faecalis strains tested in this study, and 39\% for E.faecium. These results are in concordance with findings of other studies [27]. 
In the present study, both E.faecalis and E.faecium showed multiple-drug resistance to commonly used anti-enterococcal drugs. E.faecium was found more resistant, as compared to E.faecalis. Of particular note was the emergence of concomitant resistance to 6 antimicrobials in almost $50 \%$ of the E.faecium isolates. The data presented in this paper indicate an increase of prevalence of multidrug-resistant E. faecium in our hospital. The experience from countries with high prevalence of VRE shows that an increase in VRE rates occurs several years after establishment of (vancomycin-susceptible) hospital-acquired E.faecium clonal types in hospital environment $[3,4,21]$. Although the circulation of such strains observed in our hospital, acquired resistance to vancomycin has not been established yet. This finding reflects the diverse situation regarding the VRE in Europe with prevalence ranging from $<1 \%$ to $>40 \%$ [3]. It is well known that many factors (antibiotic consumption, colonization pressure and

\section{References}

1. Leclercq R. Enterococci acquire new kinds of resistance. Clin Infect Dis. 1997;24(1):580-4.

2. Murray BE. The life and times of the Enterococcus. Clin Microbiol Rev. 1990;3:46-65.

3. Werner G, Coque TM, Hammerum AM, Hope R, Hryniewicz W, Johnson A, et al. Emergence and spread of vancomycin resistance among enterococci in Europe. Euro Surveill. 2008; 3 (47):pii 19046.

4. Willems RJ, Bonten MJ. Glycopeptide-resistant enterococci: deciphering virulence, resistance and epidemicity. Curr Opin Infect Dis. 2007;20:38490.

5. Willems RJ, Homan W, Top J, van Santen M, Tribe $\mathrm{D}$, Manzioros X, et al. Variant esp gene as a marker of a distinct genetic lineage of vancomycinresistant Enterococcus faecium spreading in hospitals. Lancet. 2001;357:853-5.

6. Willems RJ, Top J, Van Santen M, Robinson DA, Coque TM, Baquero F, et al. Global spread of vancomycin-resistant Enterococcus faecium from distinct nosocomial genetic complex. Emerg Infect Dis. 2005;11: 821-8.

7. Leavis HL, Bonten MJ, Willems RJ. Identification of high-risk enterococcal clonal complexes: global dispersion and antibiotic resistance. Curr Opin Microbiol. 2006;9:454-60.

8. Huycke M, Sahm D, Gilmore S. Multiple-drug resistant enterococci: the nature of the problem and an agenda for the future. Emerg Infect Dis. 1998;4:1218-31 limitations of effective infection control measures) together with the clonal dissemination of certain enterococcal strains contribute to the growing endemicity and nosocomial outbreaks from VRE.

\section{Conclusion}

We conclude that a multidrug resistance among enterococci in our institution is a threatening problem, which demands appropriate control, surveillance procedures and awareness for development of glycopeptide resistance.

\section{Acknowledgment}

This study was carried out with financial support of Medical University-Pleven through University Grants Commission (Project №21/2012).

9. Keuleyan E, Sredkova M, Stefaniov I, Savov E, Borissova V, Markovska R,et al. Survey on antimicrobial resistance in enterococci from human and veterinary sources. Proceedings of the XXXIII Annual Conference of Indian Pharmacological Society; 2000 December 28-30; Gandhinagar, India.

10. Lazarova G, Kantardjiev T, Velinov C, Rachkova K, Rukanova D, Dukova L,et al. Vancomycinresistant enterococci in hospitalized patients with urinary tract infections - first report in Bulgaria. Trakia J Sci. 2005;3(4):13-4.

11. Facklam RR, Collins MD. Identification of Enterococcus species isolated from human infections by a conventional test scheme. J Clin Microbiol. 1989;27(4):731-4

12. Devriese LA, Pot B, Kersters K, Lauwers S, Haesebrouck F. Acidification of methyl- $\alpha-D$ glucopiranosid: a useful test to differentiate Enterococcus casseliflavus and Enterococcus gallinarum from Enterococcus faecium species.group and from Enterococcus faecalis. J Clin Microbiol. 1996;34 (10):2607-8.

13. Enterococcus spp. Performance Standards for Antimicrobial Susceptibility Testing; TwentySecond Informational Supplement M100-S22. Clinical and Laboratory Standards Institute. 2012;32(3): 90-4.

14. Barisic Z, Punda-Polic V. Antibiotic resistance among enterococcal strains isolated from clinical specimens. Int $\mathrm{J}$ Antimicrob Agents. 2000;16(1):65-8. 
15. Sader HS, Moet GJ, Jones RN. Update on the in vitro activity of daptomycin tested against 17,193 Gram-positive bacteria isolated from European Medical Centers (2005-2007). J Chemother. 2009;21(5):500-6.

16. Zouain MG, Araj GF. Antimicrobial resistance of enterococci in Lebanon. Int $\mathrm{J}$ Antimicrob Agents. 2001;17(3):209-13.

17. Enterococci. Annual report of the European Antimicrobial Resistance Surveillance Network. (EARS-Net). Stockholm, ECDC, 2010. p. 33-7.

18. Aznar J, Lepe JA, Dowzicky M. Antimicrobial susceptibility among E. faecalis and E. faecium from France, Germany, Italy, Spain and the UK (T.E.S.T. Surveillance Study,2004-2009) J Chemother. 2012;24(2):74-80.

19. Torell E, Cars O, Olsson-Lijequist B, Hoffman B, Lindbäck J, Burman LG and The Enterococcal Study Group. Near absence of vancomycinresistant enterococci but high carriage rates of quinolones-resistant ampicillin-resistant enterococci among hospitalized patients and nonhospitalized individuals in Sweden. J Clin Microbiol. 1999;37:3509-13.

20. Torell E, Cars O, Hambraeus A. Ampicillinresistant enterococci in a Swedish University Hospital; nosocomial spread and risk-factors for infection. Scand J Infect Dis. 2001;33: 182-7.

21. Leavis HL, Willems RJ, Top J, Bonten MJ. Highlevel ciprofloxacin resistance from point mutations in $g y r A$ and $\operatorname{par} C$ confined to global hospital-adapted clonal lineage $\mathrm{CC} 17$ of Enterococcus faecium. J Clin Microbiol. 2006;44: 1059-64.

22. Torell E, Kuhn I, Olsson-Liljequist B, Haeggman S, Hoffman B-M, Lindahl C, Burman LG. Clonality among ampicillin-resistant Enterococcus faecium isolates in Sweden and relationship with ciprofloxacin resistance. Clin Microbiol Infect. 2003;9:1011-9.

23. Shouten MA, Voss A, Hoogkamp-Korstanje JA. Antimicrobial susceptibility patterns of enterococci causing infections in Europe. The European VRE Study Group. Antimicrob Agents Chemother. 1999;43:2542-6.
24. Hallgren A, Abednazari H, Ekdahl C, Hanberger $\mathrm{H}$, Nilsson M, Samuelssona A, at al. Antimicrobial susceptibility patterns of enterococci in intensive care units in Sweden evaluated by different MIC breakpoint systems. J Antimicrob Chemother. 2001;48:53-62.

25. Sredkova M. Resistance to penicillins, aminoglycosides and vancomycin in enterococci isolated from clinical specimens. Infectology. 1999,36(2):18-21. [In Bulgarian].

26. Thapa B, Tattawasart U, Manjai A, Chantarasuk Y. Antimicrobial resistance and species prevalence of eterococcal isolates in Srinagarind Hospital, Northeastern Thailand. KKU Res J (GS). 2012;7 (4):97-107.

27. Butcu M, Akcay SS, Inan AS, Aksaray S, Engin DO, Calisici G. In vitro susceptibility of enterococci strains isolated from urine samples to fosfomycin and other antibiotics. J Infect Chemother. 2011;17(4):575-8.

28. Salem-Bekhit MM, Moussa I, Muharram MM, Alanazy FK, Hefni HM. Prevalence and antimicrobial resistance pattern of multidrugresistant entrerococci isolated from clinical specimens. Indian J Med Microbiol. 2012;30(1):44-51.

29. Frazzon A, Gama BA, Hermes V, Bierhals CG, Pereira RI, Guedes AG et al. Prevalence of antimicrobial resistance and molecular characterization of tetracycline resistance mediated by tet (M) and tet (L) genes in Enterococcus spp. isolated from food in Southern Brasil. World J Microbiol Biotechnol. 2010; 26; 365-70.

30. Quinones D, Goni P, Rubio MC, Duran E, GomezLus R. Enterococcus spp. isolated from Cuba; species frequency of occurrence and antimicrobial susceptibility profile. Diagn Microbiol Infect Dis. 2005;51(1):63-7.

31. Zhanel GG, Hoban DJ, Karlowsky JA. Nitrofurantoin is active against vancomycinresistant enterococci. Antimicrob Agents Chemother. 2001;45(1):324-6. 